\title{
APPROACH TO THE PROBLEM OF BIO-INSPIRED ROBOTIC GRIPPER DESIGNING
}

The article with its content belongs to the field of bio-inspired robotics. This main focus is aimed at the problem of a specialised human-like robotic gripper designing. The article presents our approaches to solution of the chosen problems of multifunctional robotic grippers designing on the example of a proposed 3D model of three-fingered bio-robotic gripper. Attention is devoted to utilisation of CAD/CAE tools for exploring characteristics of the design using computer simulation.

Keywords: Bio-inspired robots, object grasping, computer simulation, CAD/CAE, contact analyses.

\section{Introduction}

Biologically inspired robotic subsystems - robotic hands are becoming more and more popular not only as rehabilitation devices but also as the dextrous ones for human-like manipulation with objects. Namely, the three-fingered robotic grippers as a robot arm working tool can find application in industrial field too. Automated workplaces have often required utilising robots that are able to response to changes in product production flexibly. According to the flexibility required level it can be achieved by utilisation of a robot's multiple grasping end effector, a system of automated exchange of robotic grippers or a specialised biorobotic gripper that is able to grasp objects of different types similarly to human-like way. Kinematics, actuation and control of bio-robotic grippers are more complicated than it is in case of standard industrial grippers. Therefore, the development of these special robotic subsystems design requires a special approach and application of modern methods and means of computer simulation. The present research in the field of multifunctional robotic hands/grippers development is aimed at the problem of their virtual models grasping ability exploration [1] and [2]. Novelty in robotic hands/grippers designing requires creative and innovative approach.

A multifunctional - bio-robotic gripper is a special technical system. In general, the design methodology of this system involves algorithmised procedures of technical creative work most of all. Creative technical work deals with the solution of tasks with unknown resolution procedure [3] and [4]. TRIZ as one of the worldwide applied methods suitable for solution of this type of tasks, is based on logic, data and research, not intuition approach to a problem solution. "TRIZ brings repeatability, predictability, and reliability to the problem-solving process with its structured and algorithmic approach". It offers the means that support abilities of engineers to enhance their creativity. TRIZ is based on [5]:

- the regularities of objectively existing tendencies of the technical systems development,

- and principles for the technical contradictions overcoming.

- The procedure of TRIZ methodology application in process of technical system development consists of the next steps [6]:

- a preparatory stage (the choice of the object of innovation, specification of the project team, etc.),

- an information stage (a collection of all important information about innovated object, marketing, etc.),

- a function-cost analysis of innovated object - FNA (finding answers to the questions what (?) and why (?) should be improved in a technical system),

- an algorithmization of innovative solutions - ARIZ (specification and solution of technical and physical contradictions),

- a verification stage (choice and verification of the best solution),

- a stage of construction and the proposals testing with support of $\mathrm{CAD} / \mathrm{CAM} / \mathrm{CAE}$.

Utilisation of simulation tools of appropriate $\mathrm{CAD} / \mathrm{CAE}$ system enables to obtain information how the chosen kinematic parameters and construction influence the robotic hand ability to grasp different objects relatively fast and to optimize its constructional design for required result still before its prototype production [7].

\footnotetext{
* ${ }^{1}$ Darina Kumicakova, ${ }^{2} \mathrm{Zdenek}$ Konecny

${ }^{1}$ Department of Automation and Production Systems, Faculty of Mechanical Engineering, University of Zilina, Slovakia

${ }^{2}$ Department of Robotics, Faculty of Mechanical Engineering, VSB - Technical University of Ostrava Ostrava-Poruba, Czech Republic

E-mail: darina.kumicakova@fstroj.uniza.sk
} 
The paper presents application of TRIZ methodology principles and utilisation of simulation tools of systems creo parametric and MSC ADAMS in the process of a 3D model of three-fingered bio-robotic gripper designing that was proposed for purposes of research possibilities of the object grasping stability evaluation by simulation in real time.

\section{Description of the bio-robotic gripper design}

The latest $3^{\text {rd }}$ version of bio-robotic gripper's design consists of three identical fingers that are placed at the palm of lightened construction - see Fig. 1a. Two fingers (Finger1 and Finger 2) are mounted at the gripper's palm in opposite to Finger 3. Every finger has three joints and 4DOF $(2+1+1)$. Movement of every finger is activated separately through a motion transmission system. Movement of proximal and medial links of every finger for the biorobotic gripper closing/opening is activated directly by tensile forces acting in bendable steel plaited wires - cables. Movement of each of these links is controlled by own pair of cables. Movement of distal links is activated indirectly through the rigid rods depending on the proximal links movement.

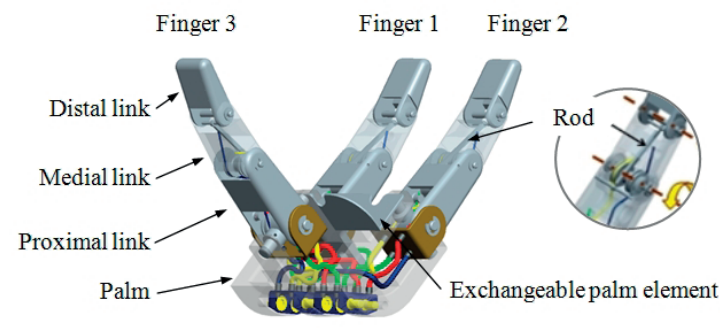

a)

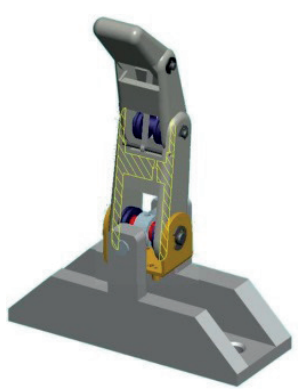

b)

Fig. 1 Design of the $3^{\text {rd }}$ version of bio-robotic gripper: a) 3D model (creo parametric 5); b) an innovated construction of the finger.
The new concept of bio-robotic gripper was created on the base of TRIZ methodology principles. Two parameters from 39 Features of Altshuller's Contradiction Matrix were chosen:

1. “35. Adaptability or versatility": This parameter represents a main requirement in the process of multifunctional robotic hands designing. But the solution of this one leads to the technical system complexity increase. It means that high requirements will be asked to specify the interaction among the technical system subsystems and their control too. Therefore, the second - worsening parameter was chosen:

2. "33. Ease of operation": the movements control.

Four principles (No.: "1.”, "15.”, "16.” and "34.”) were chosen on the base of analysis of TRIZ 40 principles [8] successfully applied to overcome the contradictions. The offered solutions have brought a new view at the bio-robotic gripper's finger construction. The new construction element was added to the finger's $1^{\text {st }}$ joint (application of principle “1." Segmentation). Applications of the principles: "15. Dynamics", 16. Partial or excessive actions and "34. Discarding and recovering" have led to a change of cables guiding towards to the finger medial link and an elimination of pulleys as the source of redundant forces that acted between the finger's distal and medial links. This new concept of finger (Fig. 1b) was applied for creation of the bio-robotic gripper's $3^{\text {rd }}$ construction.

\section{Simulation model of bio-robotic gripper}

The basic problem of the bio-robotic gripper's mechanism simulation resulted from a large number of both degrees of freedom and members of the kinematic chain whose movement must be controlled. The finger's mechanical structure represents an open kinematic chain for which the physical parameters and their boundary conditions were not precisely defined. Therefore, the principle of bionics and heuristic approach based on empiricism and analogy with human hands will be used for the motion simulation [9].

The first phase of the flexible components simulation was focused on computer modelling of plaited wires (cables) as the flexible elements which constitute the actuation and transformation mechanism of the bio-robotic gripper's propulsion subsystem. Two approaches were applied in this phase.

The idea of implementing the necessary simulations was to use Pro/Engineer WF5 and apply alternative approaches to modelling and simulation of mentioned cables [10]. We considered the cable as a $3 \mathrm{D}$ model with a constant length, resulting in the subsequent simulation procedures (chain method). Solution like this one represents the application of simulation based on object modelling features of the mechanism. The basic principle of testing was to simplify the real model to the theoretical one to eliminate many parameters and variables entering the process 
that could affect the test results in ways previously unpredictable. The bendable wire was modelled as a linkage consisting of $n$ cylindrical elements of constant length:

$d l_{1}=d l_{2}=\ldots=d l_{n}$

Total length of the cable model is expressed as follows:

$$
l=\sum_{i=1}^{n} d l_{i}
$$

The cable model's base element - a cylinder of diameter $0.8 \mathrm{~mm}$ was closed from the one side by convex cylindrical surface and from the second side by concave surface. These both surfaces create references to the building elements for the mutual interconnection between neighbouring elements in the chain. A trajectory of cable model was defined by the cable outlet (output) at the base, a rotating guide roller surface and point of its mounting to the proximal link. This trajectory was created in the model as "Sketch" associative curve changing its shape depending on the configuration of characteristic elements position during their mutual movement. The created simplified testing model is shown in Fig. 2. A previously considered actuator - artificial pneumatic muscle (PAM) is modelled in the proximal link motion transmission system too. The simulation model of PAM is connected with an output end of the cable model. The length and diameter of PAM were defined as parameters depending on a control parameter $P$ (a pressure inside PAM). The PAM's length was changed during the simulation with subsequent change in control parameter $P$ and by regeneration of the simulation model. It led to the cable tensing and rotational movement of the finger's proximal link execution.

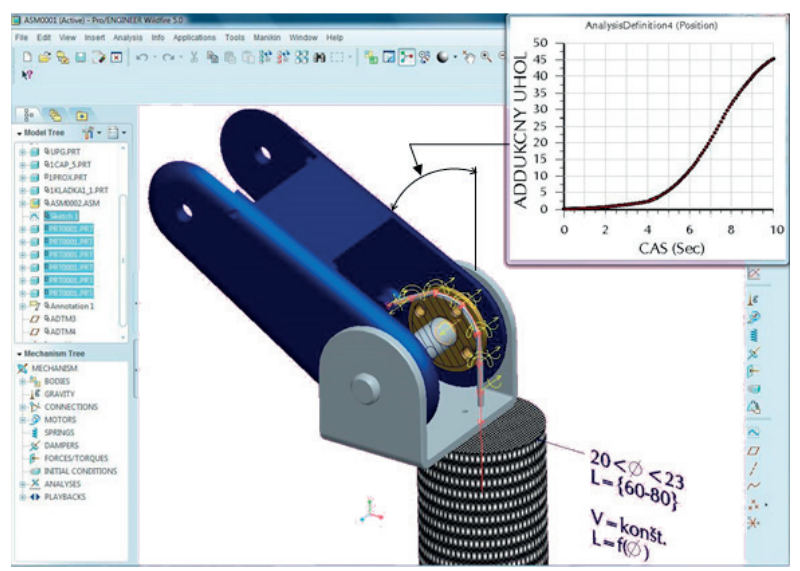

Fig. 2 Tested simulation model of the finger proximal link motion transmission system (3D model; creo parametric).

The results of the simulation experiments showed that [10]:

- the difficulty and time of simulation calculations increase in proportion to the increasing number of elements $n$. It could be caused by non-optimized calculation algorithm system for a given application and hardware performance PC.

- a simulation based on variations of parameter $P$ enables to obtain only discrete values (angular rotation and position).

The conclusion was that this approach to the motion transmission system model creation will not be suitable for exploration of the bio-robotic gripper grasping abilities by simulation in real time and will not be appropriate for the complex bio-robotic gripper mechanism creation.

The new testing simulation model was created in ADAMS/ View [11]. A pulley mechanism was proposed for a transmission simulation model creation. The cable was modelled as "immaterial" element placed between two pulleys in tangential contact points. Kinematic dependency between movement of the cable model and pulleys was defined by Rackpin Joint. In this way the tensile force acting on the cable could be transformed to the pulley's torque and conversely too. The following assumptions were adopted too:

- Tendons (cables) are ideally rigid-bodies.

- Masses of tendons (cables) and pulleys are negligible.

- Friction between a tendon (cable) and a pulley is infinitely large - a pulley transmits the torque.

- Friction between a pulley and its pin is negligible.

Proposed testing model was validated with the help of ADAMS tool. Also a correct function of the testing model was verified by dynamic simulation. Obtained results showed a direct kinematic dependency between angular rotation of medial and distal links. The finger testing model could be utilized for the bio-robotic gripper virtual model creation. Further, the tasks of 3D contacts modelling for simulation of the bio-robotic gripper grasping ability in real time in MSC ADAMS 2012 were solved [12]. The simulation experiment of a cylindrical object grasping was executed. Measured parameters were: single tensile forces acting on the proximal and medial links cables (Table 1) and values of the cables displacements $\Delta l$ - see Fig. 3 .

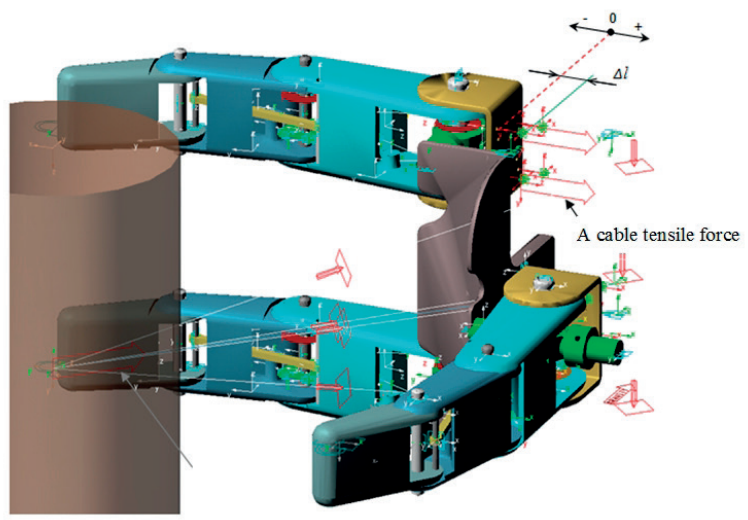

Fig. 3 Virtual model for object grasping simulation (MSC ADAMS 2012) 
Values of measured tensile forces in moment of the object stable grasping

Table 1

\begin{tabular}{|l|l|l|l|l|l|}
\hline \multirow{2}{*}{ Object: a cylinder } & \multirow{2}{*}{ Closed bio-robotic gripper } & Finger 1 & \multicolumn{2}{|l|}{ Finger 2 } & Finger 3 \\
\cline { 3 - 5 } & & \multicolumn{3}{|c|}{ Tensile forces $-\mathrm{F}_{\text {max }}(\mathrm{N})$} \\
\hline \multirow{2}{*}{ Material: wood; $\varnothing \mathrm{D}=60 \mathrm{~mm}, \mathrm{~L}=110 \mathrm{~mm}$} & Proximal link cable & 2.877 & 2.877 & 5.764 \\
\cline { 2 - 5 } & Medial link cable & 3.609 & 3.609 & 7.209 \\
\hline
\end{tabular}

Measured values of tensile forces for Fingers 1, 2 and 3 demonstrate a symmetrical configuration of forces on single fingers resulting from the steady state of forces acting at the grasped object in the moment of its stable grasping.

In addition, previously considered actuators - pneumatic artificial muscles were replaced by electric motors Maxon EC 20 including a simple transmission system (Fig. 4). The pulley (3) for fixation and reeling the cables was specially designed.

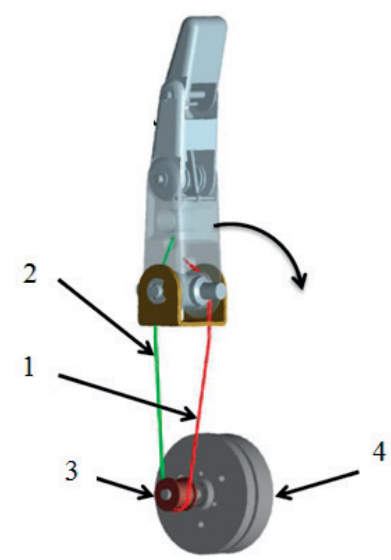

Fig. 4 Principle of a motion transmission system proposed for the proximal links movement: 1 - a cable activated on finger closing; 2 - a cable activated on finger opening; 3 - a pulley; 4 - Maxon EC 20.

\section{Analysis of an object grasping optimal conditions}

Generally, the requirements to minimize forces or torques actuating on robotic gripper and also the reducing of mechanism whole mass at a minimum are important for a robotic gripper construction These conditions are common also for a multifunctional bio-robotic gripper. An optimal value of transfer function $(U / F)$ is sought for an object grasping effective way. The transfer function is given as follows [13]:

$\frac{U}{F}=f\left(d_{1}, d_{2}, d_{3}, \ldots, d_{n}\right)$,

where:

$U$ - the value of grasping force $(\mathrm{N})$,

$F$ - the value of actuating force of the mechanism actuating system $(\mathrm{N})$

$d_{1}, d_{2}, d_{3}, \ldots, d_{n}$ - parameters defining the kinematic structure of a robotic gripper.

All known optimization methods are based on seeking the minimal value of a target function. The presented $\mathrm{CAD} / \mathrm{CAE}$ systems are able to solve a complex optimization tasks too. They integrated the different FEM methods that open new possibilities of components and assemblies analyses [14]. Contact analyses don't require calculation of reactions of mechanism single joints for a lot of cases. The primary force (or torque) is applied on

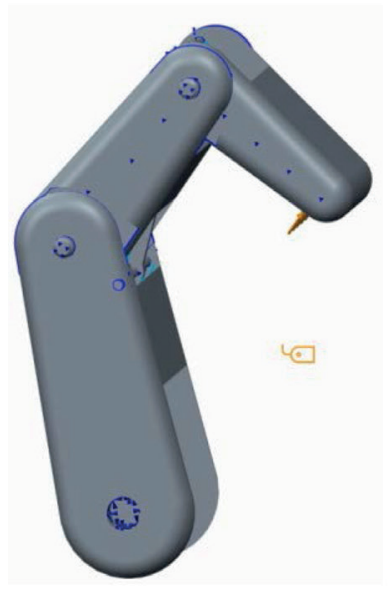

a)

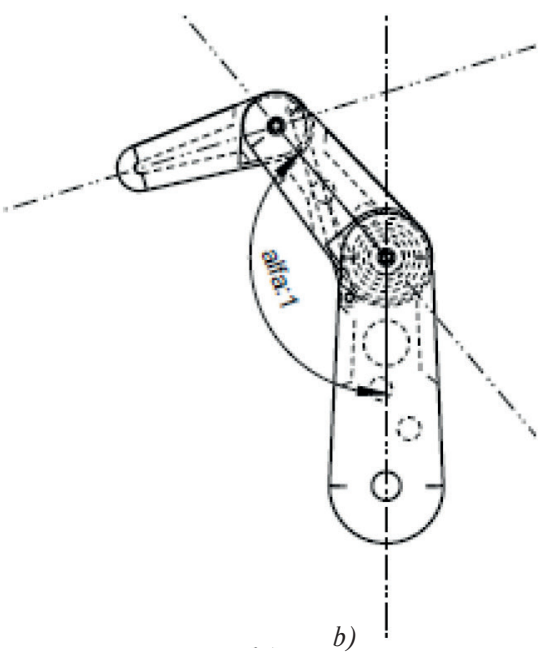

b)

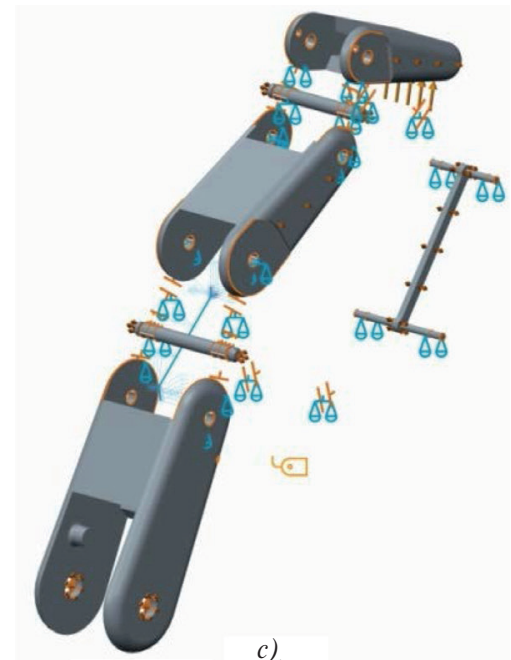

Fig. 5 Created computational model of the finger (creo 2): a) a simplified model; b) the specification of a parameter alfa:1; c) created contacts. 

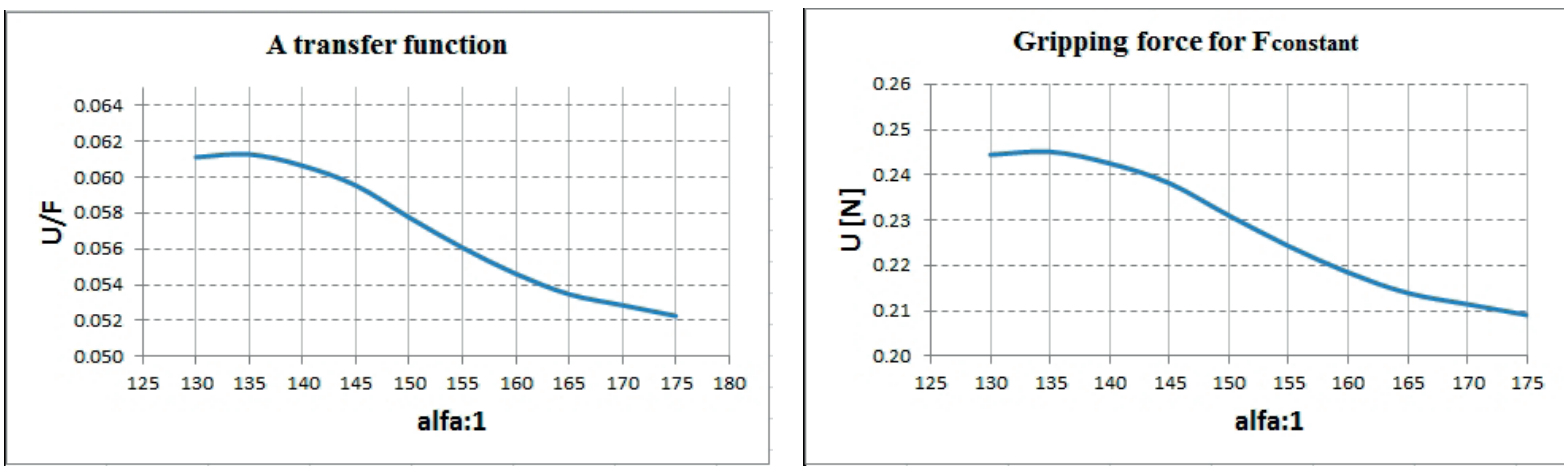

Fig. 6 Results of contact analyses (creo2)

mechanism and is analysed as a whole. Influence of responses is automatically reflected in deformations of the mechanism individual components. If the contact analyses of flexible parts are realized, the mechanism's redundancies have to be eliminated to obtain regular results of analyses [15]. A simplified computational model of the bio-robotic gripper's finger was used for the optimal transfer function seeking (Fig. 5a). The defined parameter alfa:1 (Fig. 5b) is an angle between the finger's proximal and medial links. Contact analyses were performed for the angle that was changed from $130^{\circ}$ to $175^{\circ}$. The contact model was also created (Fig. 5c).

Material properties of the computational model were defined on the base of mechanical properties of the manufactured finger prototype. Three phalanges and one pulley are manufactured from material ABS 400 by Rapid Prototyping method that is usually used for prototype parts production [16]. The remaining parts are manufactured from steel material. The cable was simulated by element of a Beam type that enables to obtain an axis force (actuating tensile force in the solved task) that was used for the transfer function calculation. The values of Table 1 were used for definition of the mechanism model loading. Results of the contact analyses show that parameter alfa: $1=135 \mathrm{deg}$ represents angle of an optimal grip of proximal and medial links (see Fig. 6).

Results of deformation analyses showed that influence of the gripping force $U$ on the distal link is minimal (Figs. 6 and 7) therefore, material ABS400 is possible to consider as isotropic material in this case.

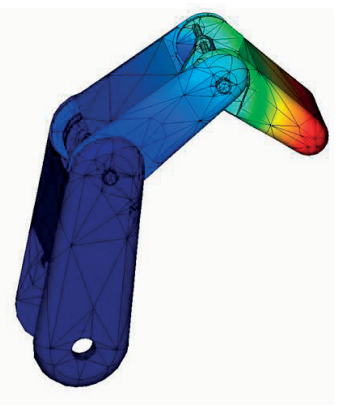

a)

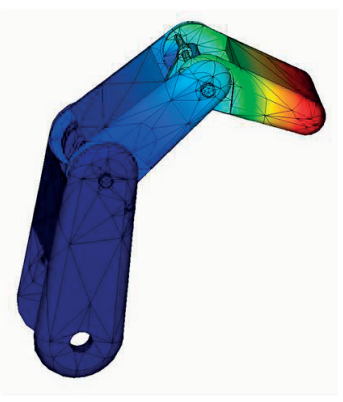

b)
Fig. 7 Deformation of the finger model: a) a model without deformation of distal link; b) a deformed model.

\section{Conclusions}

The paper presents one of research trends in robotics that is partially solved at the Department of Automation and Production Systems and Department of Robotics. The article points out approaches that have been applied in: the innovation process of $3 \mathrm{D}$ model of three-finger bio-robotic gripper, creating the simulation environment for objects robotic grasping in real time and solving of problem of utilization contact analysis for seeking the optimal grip of finger links. The future tasks will be focused on improvement of the created simulation environment and optimization of the bio-robotic gripper design.

\section{References}

[1] PRATTICHIZZO, D., TRINKLE, J. C.: Handbook of Robotics, 28, Grasping, 2008, 671-700, Springer-Verlag Berlin Heidelberg. ISBN: 978-3-540-23957-4.

[2] KURIC, I.: New Methods and Trends in Product Development and Process Planning, Academic J. of Manufacturing Engineering. Editura Politehnica - Scientific Papers, vol. 9, No. 1, 2011, 83-88, Cluj-Napoca. ISSN 1583-7904. 
[3] NAGY, S., R., LOBONTIU, M., KURIC, I., PETROVAN, A.: Innovative Development of One-Off and Small Series Products, Applied mechanics and Materials, vol. 371, 2013, 852-856. ISSN: 1662-7482.

[4] LiTVAJ, I., PONISCIAKOVA, O., STANCEKOVA, D., DRBUL, M.: Knowledge Processes and their Implementation in Small Transport Companies, 17 ${ }^{\text {th }}$ Intern. Conference on Transport Means 2013, Kaunas, October 2013, Code 102486, 153-156, ISSN: 1822-296X

[5] SKARUPA, J., MOSTYN, V.: Methods and Means of Industrial and Service Robots Designing, ${ }^{\text {st }}$ ed. (in Czech), Vienala: Kosice 2002. 190 p., ISBN 80-88922-55-0.

[6] TRIZ - IPA Slovakia - IPA Magazín - Creation and solution of innovative tasks. (in Slovak): [cit. 2014-03-14], [online] http://www. ipaslovakia.sk/sk/ipa-slovnik/triz-tvorba-a-riesenie-inovacnych-zadani.

[7] CUBONOVA, N.: Utilization of CAD/CAM System Pro/Engineer in Mechanical Engineering Industry. Proc. of $15^{\text {th }}$ intern. conference on manufacturing systems - ICMaS. Bucharest: Editura Academiei Romane, 2006, 445-448. ISSN 1842-3183.

[8] http://www.triz40.com/

[9] KUMICAKOVA, D., GORSKI, F., MILECKI, A., GRAJEWSKI, D.: Utilization of Advanced Simulation Methods for Solving of Assembly Processes Automation Partial Tasks. Manufacturing Technology: J. for Science, Research and Production, vol. 13, No. 4, 2013, 478-485. ISSN 1213-2489.

[10] JAKUBCIK, M., KUMICAKOVA, D.: Computer Aided Design of Unconventional Robotic Gripper. Intern. J. of Mechanics and Control, vol. 13, No. 1, 2012, 29-34. ISSN 1590-8844.

[11] KUMICAKOVA, D., JAKUBCIK, M.: Advanced Methods for the Bio Robotic Gripper Grasping Ability Exploration. Proc. of Intern. conference on innovative technologies IN-TECH 2013, Budapest, September 2013, 125-128, Rijeka: Faculty of Engineering University, ISBN 978-953-6326-88-4.

[12] KUMICAKOVA, D., JAKUBCIK, M.: Specialised Robotic Hand Designing and Object Grasping Simulation. Applied Mechanics and Materials, vol. 282, 2013, 90-98. ISSN 1660-9336.

[13] KONECNY, Z., KRYS, V.: CAD III - Strength Analysis (in Czech), Multimedia educational text. VSB-TU Ostrava, 2007. 198 S. ISBN 978-80-248-1513-8.

[14] SAPIETOVA, A., SAGA, M., NOVAK, P.: Multi-software Platform for Solving of Multibody Systems Synthesis, Communication Scientific Letters of the University of Zilina, vol. 14, No. 3, 2012, Zilina, 43-48, ISSN 1335-4205.

[15] KONECNY, Z.: Designing of Service Robots Nods (in Czech). Habilitation thesis, VSB-TU Ostrava, 116 p., 2009.

[16] NOVAKOVA-MARCINCINOVA, L., NOVAK-MARCINCIN, J., TOROK, J., BARNA, J.: Selected Experimental Tests of Materials used in Rapid Prototyping Area. Manufacturing Technology: J. for Science, Research and Production, vol. 13, No. 2, 2013, 220-226. ISSN 1213-2489. 